\title{
On the Relation between Inverse Factorial Series and Binomial Coefficient Series.*
}

\author{
By W. L. Ferrar.
}

Received and Read 5th June 1925.

The chief aim of this note is to investigate directly the relation between the inverse factorial series

$$
\sum_{n=0}^{\infty} \frac{a_{n+1} n !}{(x+1)(x+2) \ldots(x+n+1)}
$$

and the binomial coefficient series, or Newton's interpolation formula,

$$
(1+\Delta)^{x} \Omega(0)
$$

which may also represent $\Omega(x)$, the sum of the series (1).

The co-existence of such expansions was established by Nielsen, Handbuch der Theorie der Gammafunktion (1906), in his work on integrals of the type $\int_{0}^{1} t^{x} \phi(t) d t$ (p. 125). He also, p. 247, makes a direct reference to the expansion of inverse factorial series in the form (2). The relation between (1) and integrals of the type mentioned is well known-for certain regions an equivalent integral can be found for any series (1), though the regions of convergence of the two are not necessarily the same. Moreover, Nielsen's integral $\int_{0}^{1} t^{x-1} \phi(t) d t$ is assumed to be uniformly convergent over the half plane $R(x)>0$, while the integral of the same type corresponding to an inverse factorial series does not necessarily converge over this region.

Accordingly we here examine the transformation from (1) to (2) on its own merits. Noriünd, $†$ Annales de l'Ecole Normale (3)

- Added August 10th, 1925.-Science Progress for July 1925 notes a paper by J. Horn, "Math. Zeitschrift" 21 (1924) 85.95, in which work on differential and difference equations, previously done with inverse factorial series, is carried out with binomial cotfficient series. I have been unable to consult the paper itself to soe what points in the connection between the two types of series are exemplified.

t Further references to this paper will be given as Norlünd (1923). 
40 (1923), p. 44, has pointed out the similarity between the transformations of Newton series and of inverse factorial series and concludes "il paraît intéressant de rapprocher ces deux séries l'une à l'autre" -a remark which indicates that Nielsen's reference, a short statement occupying some two or three lines, has been overlooked by later writers.

The present paper also contains a short discussion (\$5) of other series which may represent the sum of an inverse factorial series.

\section{$\S 1$. Notation.}

For convenience of printing we use the following symbols

$$
(x \mid n)=\Gamma(x+1) / \Gamma(x-n+1),
$$

so that, when $n$ is a positive integer

$$
\begin{aligned}
& (x \mid n)=x(x-1) \ldots(x-n+1) \\
& (x \mid-n)=1 /(x+1)(x+2) \ldots(x+n) .
\end{aligned}
$$

Further, we use $(x, n)$ to denote $x(x-1) \ldots(x-n+1) / n$ !

\section{§1.1. Preliminary lemmas.}

If $\Delta, \mathbf{\Sigma}$ have the meanings usual in the theory of finite differences, and they are considered as operating on a function of $x$,

$$
\begin{aligned}
& \Delta(x \mid n)=n(x \mid n-1) \text { for all values of } n \\
& \Sigma(x \mid n)=(x \mid n+1) \div(n+1) \text { save when } n=-1 \\
& \Sigma(x \mid-1)=d / d x \log \Gamma(x+1)=\psi(x+1) .
\end{aligned}
$$

If the operations $\Delta, \Sigma$ are applied term by term to the series (1) the resulting series have the same region of corvergence as the original series, and their sums are $\Delta \Omega(x)$ and $\Sigma \Omega(x)$ respectively.

The proof of this is immediate on applying the theorem that "if $\Sigma b_{n}$ converges and $\Sigma\left|c_{n+1}-c_{n}\right|$ also converges, then $\Sigma b_{n} c_{n}$ is convergent," [compare Bromwich, Introduction to the Theory of Infinite Series (1908), p. 205].

\section{\$2. The expansion of $\Omega(x+h)$.}

By a known result in the theory of the hypergeometric function

$$
\begin{aligned}
1 & -\frac{h \cdot m}{1(x+m+1)}+\frac{h(h-1) m(m+1)}{1.2(x+m+1)(x+m+2)}-\ldots \\
& =F(-h, m ; x+m+1 ; 1) \\
& =\Gamma(x+m+1) \Gamma(x+h+1) / \Gamma(x+m+h+1) \Gamma(x+1)
\end{aligned}
$$

provided that $R(x+h+1)>0$. 
This result, on being divided by $(x+1)(x+2) \ldots(x+m)$, becomes $(x+h \mid-m)=(x \mid-m)-(h, 1) m(x \mid-m-1)$

$$
+(h, 2) m(m+1)(x \mid-m-2)-\ldots
$$

If then $\Omega(x)=\sum_{n=0}^{\infty} a_{n+1} n !(x \mid-n-1)$

$$
\Omega(x+h)=\sum_{n=0}^{\infty} a_{n+1} n !\left\{\sum_{r=0}^{\infty}(h, r)(-n-1 \mid r)(x \mid-n-1-r)\right\}
$$

The coefficient of $(h, r)$ in this expansion is

$$
\begin{aligned}
& \sum_{n=0}^{\infty} a_{n+1} n !(-n-1 \mid r)(x \mid-n-1-r) \\
= & \sum_{n=0}^{\infty}(-1)^{r} a_{n+1}(n+r) !(x \mid-n-1-r) \\
= & \Delta^{r} \Omega(x) .
\end{aligned}
$$

Hence provided that we can justify the rearrangement of the double series, we may write, when $R(x+h+1)>0$

$$
\Omega(x+h)=\Omega(x)+(h, 1) \Delta \Omega(x)+(h, 2) \Delta^{2} \Omega(x)+\ldots
$$

§ 2.1. Sufficient conditions for the rearrangement of the series.

The rearrangement of the double series (4) is justified if, when we put

$$
a_{n r}=(-1)^{r} a_{n+1}(n+r) !(h, r)(x \mid-n-1-r)
$$

the double series $\Sigma \Sigma a_{n r}$ is absolutely convergent.

Now, $\left|a_{n r}\right|=\mid \frac{a_{n+1} n !}{(x+1)(x+2) \ldots(x+n+1)} \cdot \frac{h(h-1) \ldots(h-r+1)}{1.2 \ldots r}$

$$
\frac{(n+1)(n+2) \ldots(n+r)}{(x+n+2) \ldots(x+n+r+1)}
$$

Since $\Gamma(-h)=\lim _{r \rightarrow \infty}\left\{1.2 \ldots(r-1) r^{-\lambda} /(-h)(-h+1) \ldots(-h+r-1)\right\}$, we can find a definite $R$ such that, when $r>R$,

$$
|(h, r)|<2\left|r^{-\lambda-1} / \Gamma(-h)\right|,
$$

and so we can find a finite $K$ such that, for every $r$,

$$
|(h, r)|<K\left|r^{-n-1} / \Gamma(-h)\right|
$$

Similarly, we can find a finite $M$ such that, for every $r$,

$$
\left|\frac{(n+1)(n+2) \ldots(n+r)}{(x+n+2) \ldots(x+n+r+1)}\right|<M\left|\Gamma(x+n+2) r^{-\infty-1} / \Gamma(n+1)\right|
$$


By the inequalities (6) and (7)

$$
\begin{aligned}
\sum_{r=0}^{\infty}\left|\frac{h(h-1) \ldots(h-r+1)(n+1) \ldots(n+r)}{1.2 \ldots r(x+n+2) \ldots(x+n+r+1)}\right| \ldots \ldots . \\
<M K|\Gamma(x+n+2) / \Gamma(-h) \Gamma(n+1)| \Sigma\left|r^{-x-n-2}\right| .
\end{aligned}
$$

If $\Sigma\left|r^{-x-h-2}\right|$ is convergent, i.e. if $R(x+h+1)>0$, a condition which has already been imposed in $\$ 2$,

$\Sigma \Sigma\left|a_{r n}\right|<A \Sigma_{(n)}\left|\frac{a_{n+1} n ! \Gamma(x+n+2)}{(x+1) \ldots(x+n+1) \Gamma(-h) \Gamma(n+1)}\right|$

where $A$ is finite.

But (9) may be written as

$$
A \Sigma_{(n)}\left|a_{n+1} \Gamma(x+1) / \Gamma(-h)\right|,
$$

and so the double series is absolutely convergent if $\Sigma a_{n+1}$ is.

Theorem I. If $\Sigma\left|a_{n+1}\right|$ is convergent and $x, x+h$ are in the convergence domain of the series (1)

$$
\Omega(x+h)=\Omega(x)+h \Delta \Omega(x)+(h, 2) \Delta^{2} \Omega(x)+\ldots
$$

whenever $R(x+h+1)>0$.

The last condition is necessary when (1) reduces to a single term.

\section{\$2.2. Alternative conditions.}

An alternative set of conditions can be obtained by a slight change in the analysis.

If $R(x) \geq-1,|(n+1)(n+2) \ldots(n+r) /(x+n+2) \ldots(x+n+r+1)|$ tends to zero or to a finite limit as $r$ tends to infinity. Hence, using (6),

$$
\text { (8) }<A \sum_{r=0}^{\infty}\left|r^{-\Lambda-1}\right|<B
$$

where $A$ and $B$ are finite numbers provided that $R(h)>0$.

Hence the sum of the double series $\Sigma \Sigma\left|a_{n r}\right|$

$$
<B \sum_{n=0}^{\infty}\left|\frac{a_{n+1} n !}{(x+1)(x+2) \ldots(x+n+1)}\right|,
$$

and is finite provided that $x$ is a point of the region of absolute convergence of the series (1). We have thus established.

Theorem II A. If $x$ is a point of the region of absolute con- 
vergence of the series $\Sigma a_{n+1} n !(x \mid-n-1)$ whose sum is denoted by $\Omega(x)$, then

$$
\Omega(x+h)=\Omega(x)+h \Delta \Omega(x)+\ldots
$$

provided that $R(x+1) \geq 0$ and $R(h)>0$.

There is, however, a further form of the theorem which removes the condition of absolute convergence. Norlünd kas shown that

if

$$
\Omega(x)=\Sigma \frac{8 ! b_{s+1}}{x(x+1) \ldots(x+s)}
$$

converges for $R(x)>\lambda$, and $a_{t+1}=b_{1}+b_{2}+\ldots+b_{1+1}$, the series

$$
\Sigma \frac{s ! a_{s+1}}{(x+1)(x+2) \ldots(x+s+1)}
$$

converges absolutely and represents $\Omega(x)$ for $R(x)>\lambda, R(x)>0$.

From this result and theorem II $A$ we have Theorem II $B$. If $R(x)>0$ and $x$ is a point of convergence of the series $\Sigma_{s} ! b_{s+1}(x-1 \mid-8-1)$ whose sum is $\Omega(x)$,

$$
\Omega(x+h)=\Omega(x)+h \Delta \Omega(x)+(h, 2) \Delta^{2} \Omega(x)+\ldots
$$

whenever $R(h)>0$.

$\$$ 2.3. The last result is easily extended to series of a somewhat more general type.

If $\theta(x)$ denote the sum of $\Sigma \frac{s ! b_{t+1}}{(x+\beta)(x+\beta+1) \ldots(x+\beta+s)} \ldots$

while $\Omega(x)$ denotes the sum of $\Sigma \frac{s ! b_{x+1}}{x(x+1) \ldots(x+8)}$,

then $\theta(x)=\Omega(x+\beta)$, and we bave

Theorem $I I C$. If $R(x+\beta)>0$ and $x$ is a point of convergence of the series (11), then

$$
\theta(x+h)=\theta(x)+h \Delta \theta(x)+\ldots
$$

whenever $R(h)>0$.

If $x$ is a point of absolute convergence of (11) the condition $R(x+\beta)>0$ may be replaced by $R(x+\beta) \geq 0$.

* Acta Math. 37 (1914) 344. Further references to this are given as Norlünd (1914). 


\section{\$2.4. Some remarks on these theorems.}

The limitations of $x$ and $h$ to certain half-planes is only to be expected from the form of the series and its region of convergence respectively. Thus in II $C$, the point $x+\beta=0$ must be excluded since it is an infinity of every term of the original series; the assumption that the inverse factorial series converges for $x$ makes it necessary that it should also converge for points to the right of $x$, but not that it should converge for points to the left of it; so that, unless we make $R(h)>0$, we cannot be certain that $\Omega(x+h)$ has a meaning.

§3. An inverse factorial series expressed as a Newton series. If we write $h-x$ for $h$ we obtain

$$
\Omega(h)=\Omega(x)+(h-x) \Delta \Omega(x)+(h-x, 2) \Delta^{2} \Omega(x)+\ldots,
$$

valid when $R(h-x)>0$. Thus, if the series

$$
\Omega(x)=\Sigma b_{s+1} 8 !(x-1 \mid-8-1)
$$

converges for $x=1$, one Newton expansion for $\Omega(x)$ is

$$
\Omega(x)=8(0)-(x-1,1) s(1)+(x-1,2) s(2)-\ldots
$$

where $s(r)=a_{1} /(r+1)+a_{9} /(r+2)+\ldots$

\$3.1. By Norlünd (1914), p. 354, Theorem V, if the series (10) is summable $(C, r)$ for $x$, whose real part is positive, then its 'sum' can also be represented by a series of the form (11) which converges for that value of $x$ when $R(\beta)>r$, and converges absolutely for that $x$ when $R(\beta)>r+1$.

Thus, if $R(h-x)>0$, the 'sum' of the inverse factorial series (10) for $x=h$ may be written (using Theorem IIC) as the convergent series

$$
\Omega(x)+(h-x) \Delta \Omega(x)+(h-x, 2) \Delta^{2} \Omega(x)+\ldots
$$

The coefficients $\Omega(x), \Delta \Omega(x), \ldots$ may here be regarded as arising from the sum $(C, r)$ of series $(10)$ or the ordinary sum of the corresponding convergent series (11)-the two 'sums' are equivalent for every $x$ when $R(x)>0$. From the foregoing we have

Theorem $I I I$. If $R(m)>0$ and an inverse factorial series is convergent or summable $(C, r)$ for $x=m$, and its sum is denoted by $\Omega(x)$, then

$$
\Omega(x)=\Omega(m)+(x-m, 1) \Delta \Omega(m)+(x-m, 2) \Delta^{2} \Omega(m)+
$$

the expansion on the right being convergent whenever $R(x-m)>0$. 
\$4. The transformations of Newton series indicated by the theory of inverse factorial series.

By theorem IV of Norlünd (1914) the sum of the series

$$
\Sigma a_{s+1} s !(x-1 \mid-s-1)
$$

may also be expressed by a convergent series of the type

$$
\Sigma b_{s+1} s !(x+\beta-1 \mid-8-1)
$$

when $R(x)>0$, and $R(\beta) \geq 0$.

The corresponding property for series of type $\Sigma b_{1}(x, s)$ is indicated by the following simple deduction from theorem III of the present paper. If $\Omega(x)$ is a function which admits an expansion both as an inverse factorial series of type (10) and as a Newton series, the Newton series being

$$
\Sigma b_{s}(x-\alpha, s) \text { where } R(\alpha)>0,
$$

then it admits an expansion $\Sigma c_{s}(x+\rho-\alpha, s)$ where $R(\rho) \geq 0$, pro. vided that the point $x=\alpha-\rho$ is a point at which the inverse factorial series is summable and $R(\alpha-\rho)>0$. Moreover the series $\Sigma b,(x-\alpha, 8)$ is known to converge only for $R(x)>R(\alpha)$, while the series $\Sigma c_{s}(x+\rho-\alpha, s)$ converges for $R(x)>R(\alpha-\rho)$. That is to say, by increasing the real part of the argument of the Newton series we can represent the function over an extended region, and thus attain any point (to the right of the origin) at which the inverse factorial series is summable. If $\Omega(x)$ admits an expansion of type (11), "to the right of $x=-\beta$ " replaces "to the right of the origin" in the last parenthesis.

The theorem for any Newton series $\Sigma b_{s}(x-1, s)$ representing any function and the extension of the domain of convergence by increasing the real part of the argument is giver by Norlund (1923), p. 36. In this case the question of summability of the inverse factorial series is irrelevant, and the domain of convergence can be increased so as to be the half plane $R(x)>\lambda$, where $\lambda$ is determined by the properties of the function to be represented by the Newton series.

Again, if (10) converges for $x=\alpha$ it converges for $x=\alpha+\rho$, $\boldsymbol{R}(\rho)>0$. Hence, if a representation of the sum of (10) as $\Sigma b_{s}(x-a, s)$ exists, valid for $R(x-a)>0$, then there is also a representation $\Sigma c_{s}(x-a-\rho, s)$ valid over the less extensive region $R(x-\alpha-\rho)>0$. A similar state of affairs occurs when the repre- 
sentation of any function by a Newton series is discussed : $c f$. Norlünd (1923), p. 36.

$\$ 4$.1. The transformation of series of the type

$$
\Omega(x)=\Sigma c_{t+1} s ! \omega^{s+1} / x(x+\omega) \ldots(x+8 \omega)
$$

by taking different values of $\omega$, real and positive, is less helpful in indicating the corresponding transformations of Newton series.

We know * that if a function, satisfying certain conditions, admits an expansion of type (14) for any real and positive value of $\omega$, there is a number $\theta$ such that the expansion of type (14) is possible when $\omega>\theta$, but not possible when $\omega<\theta$.

Suppose now that the series (14) is convergent for $x=\alpha \omega$ where $R(\alpha)>0$. If we represent $\Omega(x)$ by a series of the same type with $\omega$ replaced by $\omega_{1}$, where $\omega_{1}>\omega$, then the new series also converges for $x=\alpha \omega$. But the point $\alpha \omega_{1}$ is to the right of the point $\alpha \omega$ and so the new series converges for $x=\alpha \omega_{1}$.

$$
\Omega(x \omega)=\Sigma c_{s+1} s ! / x(x+1) \ldots(x+s)
$$

where, by our hypothesis, the series on the right converges for $x=\alpha$.

Hence there is a convergent expansion of the form

$$
\Omega(x \omega)=\Sigma n_{s}(x-a, s) \text {, convergent for } R(x-\alpha)>0 \text {; }
$$

and so, $\Omega(x)=\Sigma m_{\iota}(x-\alpha \omega)\{x-(\alpha+1) \omega\} \ldots\{x-(\alpha+s-1) \omega\}, \ldots(15)$ the last series being convergent for $R(x-\alpha \omega)>0$.

We may in the factorial series representation decrease $\omega$, but not beyond the point at which that series remains convergent for $x=\alpha \omega$. Thus we may, down to a point imposed by the factorial series representation, decrease $\omega$ in the Newton series (15). In the theory of Newton series representing any function, the factorial series representation is irrelevant and we may decrease $\omega$ as much as we please provided that $0<\omega . \dagger$

Moreover the region of convergence of (15), namely, the halfplane $R(x)>R(\alpha \omega)$, is increased by decreasing $\omega$, a fact which continues to be in evidence when the more general question is broached.

- NoRlönd (1914), Theorem VII, 361.

† NorLÜ̃ (1923), 43. 
On the other hand, the theory of inverse factorial series does not indicate the existence of a number $\gamma$ such that the Newton series representation of type (15) ceases to be possible when $\omega>\gamma$. In the general discussion of Newton series such a number appears, and only under special hypotheses is this number $+\infty$. The Newton series representing functions which may also be represented by inverse factorial series come under these special hypotheses. For, as we have seen, an increase in $\omega$ still leaves aw a point of convergence of the inverse factorial series and so leaves the expression (15) valid, though over a less extensive region.

\$5. A series which may represent the analytical continuation of series (1).

The series (1), having an abscissa of convergence $\lambda$, defines an analytic function $\Omega(x)$ which also admits a representation

$$
\Omega(x)=\int_{0}^{1} t^{x} \phi(t) d t
$$

where $\phi(t)=\Sigma a_{s+1}(1-t)^{t}$, and is regular within $|t-1|=1$.

If the radius of convergence of this series is greater than unity the series (1) is absolutely convergent over the whole plane with the exception of points $x=-1,-2, \ldots *$ The function $\Omega(x)$ can also be analytically continued over the whole plane, with the exception of points $x=-n$, whenever $\phi(t)$ can be analytically continued over the interior of the circle $|t|=1$. In such a case, an expansion exists of the form

$$
\phi(t)=b_{1}+b_{2} t+b_{3} t^{2}+\ldots,
$$

convergent for $0 \leq t \leq 1$.

Hence, when $R(x)>0$, we have

$$
\Omega(x)=\Sigma b_{s} /(x+s)
$$

- Prncemare, Annales Sci. de l'Ecole Normale (3), 22 (1905), 50.

Bromwich, loc. cit., p. 254, Ex. 1. 
Since $\Sigma b_{\text {a }}$ converges, the series $(17)$ converges uniformly* over the whole plane except near $x=-1,-2, \ldots$. The series (1) also converges uniformly for $R(x) \geq K>\lambda \geq-1$. The two series (1) and (17) must therefore be representations of the same analytic function since their sums are equivalent over the half plane $R(x)>\lambda, R(x)>0$.

The expression (17) is valid whenever $\phi(t)$ is regular within $|t-1|=1$ and within $|t|=1$.

\$5.1. This representation of an inverse factorial series gives rise to a curious relationship with the Gauss interpolation formula.

The values of $\Omega(x) \sin \pi x / \pi$ at the points $-1,-2, \ldots$ are $-b_{1}$, $h_{2},-b_{3}, \ldots$, and are zero at the points $0,1,2, \ldots$ But (17) multiplied by $\sin \pi x / \pi$ is the Cardinal function $\dagger$ of this table of values and is thus equivelent to the corresponding Gauss interpolation formula.

We have then, sssuming the possibility of the equality (17)

$\Omega(x)$ is equivalent to the Newton formula of its own table of values) $\sin \pi x \Omega(x) / \pi$ is equivalent to the Gauss formula of its own table of values $\}^{\text {. }}$

\$5.2. The series of powers and inverse power's which represent $\Omega(x)$.

If, for $0<t \leq 1$, we denote $(1 / t) \int_{0}^{t} \phi(t) d t$ by $\phi_{1}(t)$, we have

$$
\Omega(x)=\int_{0}^{1} t^{x} \phi(t) d t=\left[t^{x+1} \phi_{1}(t)\right]_{0}^{1}-x \int_{0}^{1} t^{x} \phi_{1}(t) d t . \quad R(x)>-1 .
$$

With the assumptions of $\S 5$ the integrals involved are all convergent, both in this and when we repeat the process beginning with $\int t^{*} \phi_{1}(t) d t$.

Repeating this process, and writing

$$
\phi_{n}(t)=(1 / t D)^{n} \phi(t)
$$
250-1.

" By the test given by Hardy. Proc. Lond. Math. Soc. (2), 4 (1907),

† E. T. Whitraker, Proc. Royal Soc. Etdinburgh, XXXV., (1915), 181-194. 
where the limits of each integration are taken to be $0, t$ when $R(x)>-1$,

$$
\Omega(x)=\phi_{1}(1)-x \phi_{2}(1)+x^{2} \phi_{3}(1)-\ldots
$$

The remainder after $n$ terms consists of $x^{n}$ times a convergent integral, and the series (18) has a unit circle of convergence. The series and its circle of convergence may also be obtained by expansion of the terms of (17) and the use of Weierstrass' double series theorem (Bromwich, loc. cit., p, 253).

$$
\text { Again, if } \begin{aligned}
\psi(\tau) & =\Sigma a_{s+1} \tau \\
\Omega(x) & =\int_{0}^{1}(1-\tau)^{x} \psi(\tau) d \tau \\
& =\frac{\psi(0)}{x+1}+\frac{1}{x+1} \int_{0}^{1}(1-\tau)^{x}(1-\tau) \psi^{\prime}(\tau) d \tau .
\end{aligned}
$$

If

$$
\psi_{n}(\tau)=\{(1-\tau) d / d \tau\}^{n} \psi(\tau)
$$

$$
\Omega(x)=\frac{\phi(0)}{x+1}+\frac{\psi_{1}(0)}{(x+1)^{2}}+\ldots+\frac{1}{(x+1)^{n}} \int_{0}^{1}(1-\tau)^{x} \psi_{n}(\tau) d \tau,
$$

or, writing $\phi^{(n)}(t)=(t D)^{n} \phi(t)$ where $D \equiv d / d t, \ldots \ldots \ldots \ldots \ldots(19, a)$

$$
\Omega(x)=\frac{\phi(1)}{x+1}-\frac{\phi^{(1)}(1)}{(x+1)^{2}}+\frac{\phi^{(2)}(1)}{(x+1)^{3}} \ldots+\frac{(-1)^{n}}{(x+1)^{n}} \int_{0}^{1} t^{x} \phi^{(n)}(t) d t \ldots \ldots
$$

Moreover; any $\psi_{n}(\tau)$ is anslytic within $|\tau|=1$, and for sufficiently large values of $R(x)$ the integral is convergent; so that $(19)$ is an asymptotic expansion of $\Omega(x)$.

The way in which the operator $t D$ enters into both expansions is rather curious. 